\title{
Comparative study on Jia Pingwa and Mo Yan's narrative perspective--Taking Jia Pingwa's Ruined Capital and Mo Yan's Big Breasts \& Wide Hips as the example
}

\author{
Wei Li \\ Xi'an International University, Xi'an 710077, Shaanxi, China
}

Keywords: Jia Pingwa, Mo Yan, narrative perspective.

\begin{abstract}
Jia Pingwa's novels and Mo Yan's novels have similarities in the narrative perspective, but also different, but more than the same. Jia Pingwa's novels are mainly based on the narrative of "scattered thinking", and the use of the "third-person perspective". Mo Yan's novels use "self-centered thinking" and use "composite perspective". The novels of the two have "non - state narrative perspective", and in this way the modern civilization and human nature "alienation" made a profound criticism and reflection.
\end{abstract}

\section{Introduction}

Jia Pingwa and Mo Yan in the narrative strategy of the novel are consciously seeking change, continue to explore, their narrative art of classical Chinese literature and Western modern literature narrative art to make their novels have achieved high artistic achievements. Mo Yan won the Nobel Prize for Literature, one of the important reasons is that he in the narrative art and realistic care level to the Western literary readers to show the height of the Chinese writers reached. Jia Pingwa's novel also shows his personal artistic characteristics and height. In the novel narrative perspective, Jia Pingwa and Mo Yan's novels are different, but more than the same. Jia Pingwa's novels are mainly based on the narrative of "scattered thinking". Mo Yan's novels are mainly based on the narrative of "thinking to me". Jia Pingwa uses "third-person perspective", and Mo Yan uses "composite view". Jia Pingwa and Mo Yan's novels exist "unconventional narrative perspective", which makes their novels have the meaning of allegory and cultural reflection.

\section{Jia Pingwa 's "Scatter Thinking" Narration and the Third Person Perspective}

"Scatter thinking" is a reference to Chinese painting language. Painting perspective has a Chinese style "scattered perspective", Western style "focus perspective". Chinese-style "scattered perspective" refers to the artist's observation point is not limited to one, but can have a number of observation points, the view of the King can be painted, can be described as moving. Western style "focus perspective" refers to the artist's observation point is limited to one, the real view of the scene into the picture, outside the horizon can not be painted.

[1]Jia Pingwa by the Chinese painting "scattered perspective" effect is obvious. He talked about the novel creation, said: "According to Western painting techniques, the focus of perspective, this approach I do not use, multi-use is a scatter perspective, is the skills of Chinese painting, Chinese painting performance of non-focus, Light, time and space is not strict, Meilan bamboo chrysanthemum can be the same scene. View angle may wish to change, or look at the height from the height, or from the big point to see, or turn over. "[1] 
Jia Pingwa applied the "Scatter" in the painting to the novel writing, forming the narrative of "scatter", that is, the omniscient narrative of the fixed observation angle, that is, "zero focus" or "no focus" , To avoid the linear narrative of the plane, broaden the narrative and description of the spirit behind the dimension. To Jia Pingwa's Ruined capital, for example, the text describes the last century at the end of the social transformation of urban culture decadent, in order to vent the sadness of this world. Jia Pingwa uses the "scattered thinking" narrative, describes the circle of Zhuang as the center of the formation of the circle, including different levels of people, such as artists Wang Ximian, calligrapher Gong Jingyuan, head of the band Ruanzhi Fei, there are literary and art research researcher Meng Yunfang, Magazine reporter Zhou Min, Qing Xu Temple master Huiming, as well as the various colors of women Niu Yueqing, Tang Waner, Liu Yue, A Can, and so on; In addition, also described the tattered old man and was "alienated" animal cattle. Different focus objects bear different narrative functions. "Zhuang Zhidie's social circle" shows the decadence of urban culture, is the object of criticism. The rumor of "old man" is the criticism from the bottom of the folk. A large number of monologues of "cow" with human thought are from the observation, experience, and sentiment of the "cow", which criticizes the city civilization and has deep concern about the degradation of mankind. Different focus objects implied different meaning connotation, thus increasing the depth of the text, breadth.

"Scattered thinking narrative" often builds text with third-person perspective. "Third Person Perspective" has a full view and limited perspective. Ruined capital mostly uses the third person omniscient perspective description of characters, such as: Zhuang Zhidie butter, Niu Yueqing guests at home hospitality scene description: "Niu Yueqing opened the dining room room, Meng Yun room put eight cool eight hot, four dirty Four elements, all kinds of water and wine drinks, greeted the people face clean hands are attended ...... Zhuang Zhidie first hit Wang Xi-min wife's cup, and then touched Xia Jie's cup, followed by Zhou Min, Tang Waner, Zhao Jing five, Finally, Liu month ... ... "[2]. Sometimes the text is also converted to the character of the limited perspective, such as Zhou Min first to see the village of butterflies and can not see the scene. At that time, the village of the butterfly was present, because the narrator used the limited view, from the perspective of Zhou Min to observe, Zhuang Zhidie was replaced by "the man", the text describes: Zhou Min "to the gate, see many people There is a man in the yard out of the shoes, head is not high, hair chaos, wearing a black sweatshirt, the former heart back All printed with yellow phonetic alphabet ... ... that person smiled and patted his stomach, he went to the bottom of the den under the mouth, the mouth of the nipple hand squeeze sucking up. "[2] implied author selected limited view that only from Zhou Min's perspective to describe, thus forming a narrative suspense and fun. It can be seen that the narrative strategy of Ruined capital not only allows the reader to understand the "waste" world from the perspective of the omniscient narrator from the perspective of the omniscient narrator, but also observe the "waste" from the perspective of a single angle "Waste".

\section{Mo Yan's Self-centered thinking narrative and compound person perspective}

Self-centred thinking is a kind of thinking type with strong subjectivity and mythical tendency. The thinking subject often uses the self as the center to observe, understand and evaluate the object, and this way of thinking is applied to the creation of the novel "My thinking to the mind", that is, the writer in the performance of the external world, usually with the aesthetic value of self as the standard of evaluation, my thoughts are often reflected through the object to the performance, including my subjective experience, sentiment, emotion, etc. The narrative of "I thought to thought" often constructs the text from the perspective of the first person, and the first person narrates the narrative perspective, which gives the reader what it is "I", which makes the text highly artistic, In the cordial, sincere and frank narrative, the subjectivity and lyricity of "I" are manifested....

From the perspective of "Self-centred thinking" narrative to examine Mo Yan's ig Breasts \& Wide Hips, we can see the text of a total of eight volumes (including volume outside the volume of missing missing), the first volume using the third person perspective, the second volume to the sixth volume 
One-person perspective, sometimes using the third-person perspective, the seventh and eighth volumes alternately using the first-person perspective, the "first-person" perspective and the third-person perspective. This will form a unique "complex type of people perspective", the "complex type of perspective" is based on the first person perspective.

In the narrative perspective of the first person "I", the text adopts the retrospective perspective in the narrative of the first person's hero, the experience perspective in the first person narration, the former is the external perspective, the latter is the inner perspective. The story of "I" and the story of "I" formed to see and be seen, narrated and narrated dual identity, therefore, more highlights my subjectivity and lyric, with "I thought" narrative, at the same time The narrative horizon is also more open.

In Big Breasts \& Wide Hips, in the first person perspective, the life experiences of the infants, children, juveniles, young people and middle-aged people in the same period have been described in the first-person perspective, and the rise and fall of the Shangguan family in the past century has been projected. The situation changes. "I" is suffering from serious "melancholy disease", although it is already adult, but in reality it is "giant baby", weak and incompetent, trace the birth and growth of the various periods, "I" features exactly the twentieth century and half Colonial culture and the characteristics of postcolonial culture coincide, the symbolic meaning of "I" shows the author's questioning and reflection on the modern culture formed by the fusion and conflict of things. Another important image is the mother in Big Breasts \& Wide Hips. Mother from the folk, she used folk thinking, way, the concept of things to deal with, she experienced human suffering, but to stimulate a stronger vitality, Mo Yan said that she is a symbol of the earth. "I" and the mother of these two images, all embodies the implicit author strong subjectivity, because when implied author and the text of the "I" close or overlap, as long as the character of the law of development, the reader is generally difficult Perceived, "I" can become implied author's endorsement.

"Like the first person" narrative perspective refers to the use of the characters in the seventh volume of the title "grandfather" "grandmother" "mother" and so on, this is still "I" retrospective perspective, is "I" narrative voice, For "my grandfather" "my grandmother" "my mother". This narrative perspective, which is similar to the first person, is the "first person" narrative perspective. Choose this perspective to highlight the "I" right to speak, full of "I" subjectivity and lyrical. It has a first-person perspective of the cordial, natural, real, there are third-person omniscient omnipotence, broaden the narrative horizon.

It can be seen, Big Breasts \& Wide Hips uses "composite perspective", creating a unique "I think" narrative art world. [3]

\section{Jia Pingwa and Mo Yan's "Unconventional Narrative Perspective"}

"Unconventional narrative perspective" refers to the perspective of stupid people, animal perspective and normal perspective of different narrative perspective. Jia Pingwa and Mo Yan two writers have used the silly people perspective and animal perspective and other "unconventional narrative perspective", which is a modernist "alienation perspective", as Kafka use "beetle perspective" and Han Shaogong use "Cubs perspective". The use of this perspective is the narrative strategy adopted by the two writers for the modernization of the "alienation" of the traditional humanistic and the way of thinking, but the frequency and purpose of the two are different. Jia Pingwa used less, and used to show his reflection of cultural distortions. For example, the alienated animal "cattle" in Ruined Capital, it looks with the appearance of cattle, but there are people's thinking, the author through its views and feel, directed at urban civilization, the modern city civilization was profound Reflection. Mo Yan on the "unconventional narrative perspective" to use more, such as Jin Tong in Big Breasts \& Wide Hips, Jin Tong is the Chinese and Western blood and culture co-bred out of the half-breed, "hybrid" is a serious " Melancholy "patients, extremely weak and incompetent, the author to irony in the formation of modern Chinese and Western collision culture, and its question and reflection. Mo Yan often in the novel, through this "unconventional narrative perspective" to express the "normal 
world" all kinds of "alienated" the existence of reflection, and its novel narrative has a cultural fiction function and cultural reflection.

In short, Jia Pingwa's "scattered narrative" and Mo Yan's "I to the narrative", the performance of the difference between the two narrative. The reason is that they are mainly reflected in their acceptance of Chinese classical literature tradition and Western contemporary narratology theory and aesthetic tendencies. Jia Pingwa is very fond of Chinese classical literature tradition, and he learns more about the expression of narrative art of classical Chinese literature. Mo Yan is influenced by western contemporary narratology theory and works, and created the Chinese-style "magic realism". They are concerned about modern civilization, "non-state narrative perspective" on modern civilization and human nature "alienation" made a profound criticism and reflection.

\section{Acknowledgments}

The project is a special research project of Shaanxi Province Education Department , project name: comparative study on Jia Pingwa and Mo Yan's narrative (No.: 14JK2115)

\section{References}

[1] Sun Jianxi.Prequel of Jia Pingwa(Vol.2). Guangzhou: Huacheng Publishing House, 2001. P399

[2] Jia Pingwa. Ruined capital. Beijing: Beijing Publishing House, 1993. P 96, P20-21

[3] Mo Yan. Big Breasts \& Wide Hips. Shanghai: Shanghai Literature and Art Publishing House, 2012. 